\title{
Revista

\section{Dificuldades vivenciadas pelo enfermeiro na utilização de indicadores de processos}

\author{
Difficulties experienced by nurses in the use of process indicators \\ Dificultades experimentadas por el enfermero en el uso de indicadores de procesos
}

\section{Priscilla Izabella Fonseca Barros de Menezes', Maria D 'Innocenzo"}

' Universidade Estadual de Montes Claros, Curso de Graduação em Enfermagem. Montes Claros-MG, Brasil.

"Universidade Federal de São Paulo, Departamento de Enfermagem,

Disciplina de Enfermagem em Saúde Pública e Administração Aplicada à Enfermagem. São Paulo-SP, Brasil.

\author{
Submissão: 19-09-2011 Aprovação: 25-05-2013
}

\section{RESUMO}

A pesquisa investigou as dificuldades dos enfermeiros da Santa Casa de Montes Claros-MG em utilizar indicadores para avaliar a qualidade dos resultados dos processos assistenciais. Trata-se de estudo transversal de abordagem quantitativa realizada por meio de questionário aplicado aos enfermeiros que obedeceram aos critérios de inclusão. Como resultado encontrou-se que $94,9 \%$ dos participantes relataram dificuldades para utilizar indicadores de processo, sendo $50 \%$ graduados há menos de três anos e apenas 30\% receberam informações sobre o assunto na graduação. Entre as dificuldades encontraram-se o acúmulo de atividades privativas $(61,5 \%)$, falta de conhecimento teórico e prático sobre o tema $(46,2 \%)$, pouco conhecimento da Gestão por Processo $(43,6 \%)$, pouco envolvimento da equipe na coleta de dados $(30,8 \%)$, dentre outros. Conclui-se ser importante refletir sobre as atividades privativas do enfermeiro dentro das unidades de saúde e a reorganização do seu processo de trabalho, além da inserção do tema nos cursos de graduação em enfermagem.

Descritores: Indicadores de Serviços; Gestão da Qualidade; Serviços de Enfermagem.

\section{ABSTRACT}

The research has investigated the difficulties that nurses from "Santa Casa" Hospital in Montes Claros-MG have had in using indicators to analyze the quality of care processes results. A transversal study of quantitative approach was carried out, with a questionnaire being applied to nurses who have obeyed the inclusion criteria. As a result, it was showed that $94,9 \%$ of the participants reported difficulties in using process indicators, considering that $50 \%$ of them got graduated less than three years ago and just 30\% received information about the subject during the course. Among the difficulties, the rise of privative activities $(61,5 \%)$, lack of theoretical and practical knowledge on the subject $(46,2 \%)$, little knowledge about Process Management $(43,6 \%)$, little involvement of the staff on the data collection $(30,8 \%)$, among others. Thus, it was concluded how important it is the reflection on the nurse's privative activities inside the health facilities and readjustment of their work process, besides the insertion of the subject into nursing under graduation and graduation courses.

Key words: Indicators of health Services; Quality Management; Nursing Services.

\section{RESUMEN}

Eso trabajo ha investigado las dificultades que los enfermeros del Hospital "Santa Casa" en Montes Claros-MG tienen en utilizar indicadores para analizar la calidad de los resultados de los procesos asistenciales. Tratase de un estudio transversal de abordaje cuantitativo realizado a través de un cuestionario aplicado a los enfermeros que obedecieron a los criterios de inclusión. Como resultado, se encontró que $94,9 \%$ de los participantes relataron dificultades en utilizar indicadores de proceso, considerando que $50 \%$ graduaron hace menos de tres años y sólo 30\% tuvieran informaciones sobre el asunto durante el curso. Entre las dificultades, se encontraron la acumulación de actividades individuales $(61,5 \%)$, falta de conocimiento teórico y práctico acerca del asunto $(46,2 \%)$, poco conocimiento sobre Gestión por Proceso $(43,6 \%)$, poco envolvimiento del equipo en la recogida de datos $(30,8 \%)$, y otros más. Así, se considera importante reflectar sobre las actividades individuales del enfermero dentro de las unidades de salud y la reorganización de su proceso de trabajo, además de la inserción del asunto en los cursos de graduación en enfermería.

Palabras clave: Indicadores de Servicios; Gestión de Calidad; Servicios de Enfermería.

\section{AUTOR CORRESPONDENTE Príscilla Izabella Menezes E-mail: pifbm@yahoo.com.br}




\section{INTRODUÇÃO}

Atualmente, com o intuito de se manterem economicamente viáveis, os hospitais têm que se adequar aos parâmetros legais cabendo ao gestor hospitalar escolher o modelo de gestão mais apropriado à realidade da instituição. Dentre os muitos modelos gerenciais, o de Gestão pela Qualidade tem recebido atenção por destacar a qualidade no atendimento, indispensável na área da saúde, que requer na sua essência a gestão por processos baseada em informações, análises, controle e aperfeiçoamento.

É impossível trabalhar com gestão da qualidade sem a compreensão dos referenciais do Modelo Donabediano, que descreve um tripé de sustentação de qualquer organização, composto de "estrutura" (características estáveis das instituições: área física, recursos humanos, materiais, financeiros e o modelo organizacional), "processo" (conjunto de atividades desenvolvidas na produção em geral, nas relações estabelecidas entre profissionais e usuários dos serviços - desde a busca de assistência até o diagnóstico e o tratamento) e "resultado" (características dos produtos ou serviços, cuja qualidade se traduz nos efeitos na saúde do cliente e da população) ${ }^{(1)}$.

A Gestão da Qualidade tem como princípio a gestão por processos devido a sua eficiência na busca pela satisfação dos clientes e melhoria contínua de suas tarefas de trabalho, administrando-as de modo a defini-las, descrevê-las, analisá-las, documentá-las e melhorá-las permanentemente e de maneira interligada $^{(2)}$. E, para se tornar eficiente, as principais atividades e etapas do processo necessitam de constante avaliação, realizada por meio da utilização dos indicadores que traduzam a qualidade esperada nas tarefas de cada etapa do processo assistencial (2). Essa qualidade dos processos hospitalares é averiguada pela Acreditação Hospitalar através de instituições avaliadoras certificadas pela Organização Nacional de Acreditação (ONA).

Nesse cenário, o mercado de trabalho exige do enfermeiro o seu aperfeiçoamento na utilização de instrumentos de gestão, como bases ideológicas e teóricas de administração e prática do gerenciamento de recursos, no que se refere às metas da instituição, à sua equipe de trabalho, e aos seus clientes. Porém, ainda não foram produzidos modelos capazes de padronizar a gestão de informação nos hospitais, sendo preciso que cada instituição desenvolva sua capacidade e competência em utilizar os indicadores, fazendo da gestão da informação um processo dinâmico e inerente ao serviço ${ }^{(3-4)}$.

Há o entendimento da dificuldade de eleger os sistemas de avaliação, bem como os indicadores de desempenho institucional adequados para apoiar a gestão hospitalar, traduzindo a necessidade dos administradores hospitalares e profissionais da área de aprofundar os estudos sobre o assunto(5).

Com isso surgiu a necessidade de entender como o enfermeiro utiliza essa ferramenta, considerando que, no contexto em que o estudo foi realizado, a Irmandade Nossa Senhora das Mercês de Montes Claros - MG, é possível afirmar que tem sido difícil a utilização segura e adequada de indicadores na prática de enfermagem, quer pela carência de melhor qualificar os enfermeiros para esta ferramenta, quer pela necessidade de se estabelecer indicadores precisos e que sejam de fácil mensuração e análise, e que, ao mesmo tempo, possam trazer respostas para a melhoria assistencial, buscando fortalecer nesses profissionais a necessidade de se capacitar técnica e cientificamente para atingir uma maior qualidade nos serviços de enfermagem, ou que os habilite a transformar os dados coletados em informações gerenciais.

Este estudo teve por finalidade identificar a(s) dificuldade(s) vivenciada(s) pelo enfermeiro da Santa Casa de Montes Claros - MG em utilizar indicadores de processos assistenciais, sejam esses indicadores pertencentes a qualquer fase / etapa do processo. Ou seja, não é intenção do estudo abordar os tipos de indicadores e suas variáveis, mas, sim, de investigar as dificuldades inerentes à sua utilização, mapeando a informação sobre o tema recebida por esses profissionais durante a sua graduação e no mercado de trabalho, e ainda, verificar os obstáculos enfrentados para o envolvimento da equipe de enfermagem na coleta dos indicadores, fundamental para qualquer tomada de decisão para melhoria da assistência ao cliente.

\section{REVISÃO DE LITERATURA}

Atualmente é inaceitável a ideia de cuidar do outro sem qualidade, que nada mais é do que colocar o cliente em posição de destaque em todos os processos assistenciais, atendendo-o da melhor maneira possível e isso apenas se faz praticável por meio de uma interligação dos processos e da estrutura institucional, capaz de fornecer a melhor assistência ao paciente ${ }^{(6)}$.

Como qualidade é um conceito abstrato, ela vem sendo definida de diversas maneiras, de acordo com as necessidades de cada empresa e dos seus objetivos para se trabalhar dentro de padrões reconhecidos. Assim, todas as significações se complementam, representando a qualidade como um termo de excelência, de valor, de conformidade a critérios a serem seguidos e de satisfação dos clientes usuários dos serviços de saúde ${ }^{(4)}$.

A preocupação com a Gestão de Qualidade é remota, sendo que seu auge é considerado no pós-guerra de 1945, quando se viu desenvolvida nas indústrias japonesas. Vagarosamente, essa preocupação com a qualidade foi sendo absorvida pelo setor saúde no final da década de $1960^{(6)}$.

No momento presente, há vários dispositivos legais reguladores da prática assistencial, todos com objetivo comum de fornecer parâmetros e incentivos para a prestação de uma assistência segura e com qualidade pelas instituições de saúde. Merece destaque o Manual Brasileiro de Acreditação Hospitalar, constituído por um conjunto de regras, normas e procedimentos relacionados com a certificação da qualidade dos serviços prestados pelas instituições de saúde. Criado em 1998, após vários estudos dos manuais internacionais e nacionais obteve-se um consenso entre pesquisadores para se atingir os padrões de avaliação dos serviços de saúde que seriam comuns a todos eles. Um ano após, foi criada a ONA, uma organização não governamental com atuação nacional responsável por iniciar a implantação das normas técnicas e o credenciamento das instituições acreditadoras, além da 
capacitação dos avaliadores e aprimoramento da qualidade da assistência da saúde em todo o Brasil ${ }^{(7)}$.

Os dados obtidos com a utilização de um instrumento validado servem de marcadores da qualidade, pois permitem avaliações subsequentes, possibilitando os reajustes nas metas que almejam a melhor qualidade possível, melhorando atuação gerencial(8).

Assim, torna-se necessário que o enfermeiro desenvolva habilidades na área de administração em enfermagem, abandonando a visão de que gerenciar é menos importante que cuidar do paciente, uma vez que não é possível existir um cuidado desvinculado da coordenação desse processo ${ }^{(3)}$.

Ainda não há na literatura uma concordância de ideias quanto à definição e classificação dos indicadores de qualidade, mas é possível verificar uma tendência em distinguir entre as dimensões estrutura, processo e resultados da qualidade, conforme a formulação clássica de Donabedian, citada acima. E assim, realizar estudos que analisem o processo assistencial por meio de mensurações de resultados com os indicadores, que nada mais são do que "sensores que auxiliam a verificar se os objetivos propostos foram ou não alcançados" ${ }^{\prime \prime(4) 6}$.

A importância da utilização dos indicadores pelas empresas se justifica na necessidade de acompanhamento e mensuração dos seus resultados, da verificação do alinhamento desses com os objetivos da instituição, além da possibilidade de avaliação das ações planejadas para o alcance das metas ${ }^{(9)}$. Todas essas razões são aplicáveis à área hospitalar, devendo o enfermeiro deter tal conhecimento.

Os indicadores são essenciais para o processo de tomada de decisão, pois oferecem informações seguras acerca da empresa, e ao utilizá-lo o profissional da enfermagem deve ter certeza do que medir, e quais são os requisitos escolhidos para serem trabalhados ${ }^{(9)}$.

Existe uma deficiência na seleção dos indicadores pelos administradores hospitalares, pois na grande maioria dos casos, ela não é realizada consoante às estratégias da instituição, mas simplesmente utilizados conforme solicitação da Secretaria de Saúde ou pelo uso rotineiro, desconsiderando as necessidades do gestor, a literatura científica e a importância das discussões internas e experiências anteriores ${ }^{(5)}$.

A escolha dos indicadores ou a sua elaboração pressupõe determinados conhecimentos, exigindo uma sequência lógica (item de controle, maneira de expressão, tipo, fonte de informação, método, amostra, responsável, frequência, objetivo) na sua formulação, além do conhecimento dos clientes internos e externos e as suas necessidades ${ }^{(10)}$.

O Hospital da Irmandade Nossa Senhora das Mercês de Montes Claros-Santa Casa, com o objetivo de acompanhar os novos modelos de saúde, buscou reestruturação de seus serviços, em setembro de 2005, vislumbrando trabalhar com a gestão por processos, buscando intercâmbio e melhor interação entre os diversos setores e processos institucionais. O objetivo dos administradores da instituição era gerenciá-la como uma verdadeira empresa, formada por um conjunto de processos administrativos e médico-hospitalares direcionados para a "condição de saída do paciente, cuja qualidade ao final depende da efetividade e eficiência de cada um"(11).
Com a reestruturação, o hospital foi dividido em várias gerências, sendo que a Assistencial, na qual se encontra a enfermagem, foi composta por seis áreas com o objetivo de realizar um controle sistemático e eficiente da qualidade de seu processo. Essa alteração organizacional tornou inadiável a medição de desempenho e análise do ambiente interno, pois é através de medições que se controlam e analisam as ações dos colaboradores ${ }^{(12)}$, o que foi realizado em todas as unidades da instituição.

Mas, antes de iniciar o novo modelo de gestão e a consequente utilização dos indicadores, os gestores realizaram uma série de treinamentos ministrados por uma empresa credenciada pela ONA como Instituição Acreditadora responsável pela Avaliação e Certificação de Qualidade das Organizações Prestadoras de Serviços de Saúde.

A citada Instituição Acreditadora, após realizar um diagnóstico situacional da Santa Casa, baseado no Manual de Acreditação Hospitalar da ONA, executou treinamentos para todos os gerentes, enfermeiros e chefes de clínicas médicas com foco principal no desenvolvimento da qualidade e segurança do atendimento dos clientes/pacientes, obtidos por meio da organização e interligação de todos os processos internos hospitalares, sendo assim introduzidos na instituição os princípios básicos para se trabalhar com indicadores.

Posteriormente, os treinamentos foram organizados e conduzidos pelo Escritório da Qualidade e as dúvidas desse sanadas com consultores. Porém, é sabido que na maioria dos treinamentos e consultorias, são repassados modelos teóricos ideais para ser utilizado, o modus operandi é deixado totalmente sob a responsabilidade da empresa contratante surgindo, assim, as dificuldades de utilização prática das ferramentas.

Findados os treinamentos, cada gestor reuniu-se com sua equipe de trabalho para delineamento do seu processo e definição dos indicadores principais para trabalharem a qualidade e melhoria de suas tarefas e atividades. Diante dessa realidade, os enfermeiros necessitaram aprender a trabalhar com indicadores, surgindo dificuldades para a incorporação dessa prática na ação, quer assistencial, quer gerencial do enfermeiro.

\section{METODOLOGIA}

Trata-se de um estudo exploratório e descritivo, de abordagem quantitativa. A pesquisa descritiva consiste na descoberta e observação de fenômenos procurando descrevê-los, classificá-los e observá-los. Por conseguinte, esse tipo de pesquisa descreve as características de determinada população ou fenômeno ou estabelecimento de relações entre variáveis e, em alguns casos, a natureza das relações ${ }^{(13-14)}$.

O cenário de estudo desta pesquisa foi a Irmandade Nossa Senhora das Mercês de Montes Claros - Santa Casa, na cidade de Montes Claros - MG, caracterizada como um hospital geral de referência em toda região do Norte de Minas Gerais e Sul do Estado da Bahia composto por 320 leitos de cunho filantrópico sem fins lucrativos, 50 cargos e mais de 1.100 funcionários, sendo que desse total, 65\% são da área 
de enfermagem que possui 06 enfermeiras que ocupam cargo gerencial e 36 atuantes diretos da assistência ao cliente, totalizando 42 enfermeiras(os).

A população da pesquisa foi constituída por 40 enfermeiros que atuam em todos os setores assistenciais e unidades gerenciais, prestadores diretos ou indiretos de cuidados ao paciente e que, concomitantemente, necessitam utilizar indicadores para avaliação de resultados dos processos assistenciais.

O instrumento para a coleta de dados constituiu-se de um questionário que englobava questões fechadas e alguns itens abertos que permitiram registrar as opiniões dos enfermeiros dentro do contexto. As perguntas foram respondidas com a mesma ordem e opção de resposta pelos participantes ${ }^{(15)}$. É importante ressaltar que, para verificar a viabilidade do questionário, o mesmo foi previamente testado por meio de um pré-teste antes da coleta de dados, sendo os erros encontrados corrigidos através da escala de Likert.

A coleta de dados foi realizada pela própria autora, no período de junho a agosto de 2009, no setor de trabalho de cada participante, onde os pesquisados foram instruídos sobre os objetivos e importância da pesquisa, assim como a forma de preenchimento do questionário.

O estudo foi submetido aos Comitês de Ética da Universidade Estadual de Montes Claros e da Universidade Federal de São Paulo, que avaliou e aprovou a sua realização, com registro no Sisnep com CAAE n ${ }^{\circ} 1887 / 08$. Todos os sujeitos assinaram o Termo de Consentimento Livre e Esclarecido.

Os dados foram armazenados em bancos de dados, tabulados em índices percentuais e testes paramétricos com o auxílio de profissional da área. A análise dos dados foi dividida em duas etapas: descrição da população dos enfermeiros e análise das dificuldades de utilização dos indicadores por esses profissionais. Na primeira, as variáveis numéricas foram descritas por meio de medidas de tendência central e de variabilidade (média, mediana e desvio-padrão) e as variáveis categóricas (qualitativas) trabalhadas através de frequências absolutas e relativas. São exemplos dessas variáveis: gênero, idade, anos de conclusão da graduação, tipo de faculdade (pública ou privada), tempo de trabalho e cargo ocupado na Instituição e titulação (Especialista Mestre ou Doutor). Já na segunda etapa, as variáveis foram agrupadas por similaridade e apresentadas em frequências relativas, por se tratar de variáveis de natureza qualitativa relacionadas às dificuldades do enfermeiro em utilizar indicadores.

Nas análises estatísticas, utilizou-se o teste não paramétrico do Qui-quadrado de Pearson e o Coeficiente de Correlação de Sperman, com nível de significância 0,05. Para tabulação, descrição e análise dos dados utilizou-se o software SPSS 14.0.

\section{RESULTADOS}

A instituição onde o estudo foi realizado possuía na época da coleta de dados 42 enfermeiros integrantes da Gerência Assistencial, sendo que dois foram excluídos do estudo por não aceitarem colaborar com a pesquisa.

Sobre o tempo de trabalho na Santa Casa, observou-se que $60,0 \%$ dos participantes da pesquisa trabalhavam na instituição há mais de um ano e menos de cinco, e que 22,5\% dos enfermeiros foram admitidos há menos de um ano, contrapondo com apenas $17,5 \%$ que estavam na instituição há mais de cinco anos.

O grupo era formado predominantemente por profissionais do sexo feminino (85\%), com tempo de formação inferior a três anos (50\%), advindos principalmente de entidades privadas de ensino superior (52\%) e recém-inseridos no mercado de trabalho. Embora 50\% dos enfermeiros fossem graduados há menos de três anos, apenas 30\% desses receberam informações sobre indicadores na graduação.

Quanto à utilização de indicadores de processo como ferramenta para se avaliar a qualidade dos resultados do processo assistencial, constatou-se que 70,0\% dos enfermeiros tiveram seu primeiro contato com indicadores na instituição onde o estudo foi realizado e os demais, ou estudaram o tema na graduação $(17,5 \%)$ ou na pós-graduação (12,5\%). Observou-se que $77,5 \%$ dos participantes da pesquisa não haviam tido nenhum tipo de contato teórico ou prático com o tema durante a graduação. Ainda, considerando todos os enfermeiros integrantes do estudo, 94,9\% relataram dificuldades para utilização de indicadores de processo. A relação entre o contato com indicadores e tempo de graduação encontra-se na Tabela 1.

Tabela 1 - Relação entre o tempo de graduação e o contato com indicadores na faculdade. Montes Claros, 2009.

\begin{tabular}{|c|c|c|c|c|}
\hline \multirow{2}{*}{\multicolumn{2}{|c|}{ Tempo de Graduação }} & \multicolumn{2}{|c|}{ Contato com indicadores na Faculdade } & \multirow{2}{*}{ Total } \\
\hline & & Sim & Não & \\
\hline \multirow{2}{*}{ Até 3 anos } & $\mathrm{n}$ & 14 & 06 & 20 \\
\hline & $\%$ & $70,0 \%$ & $30,0 \%$ & $100,0 \%$ \\
\hline \multirow{2}{*}{ Entre 3 a 6 anos } & $\mathrm{n}$ & 13 & & 13 \\
\hline & $\%$ & $100,0 \%$ & & $100,0 \%$ \\
\hline \multirow{2}{*}{ Mais de 6 anos } & $\mathrm{n}$ & 04 & 03 & 07 \\
\hline & $\%$ & $7,1 \%$ & $42,9 \%$ & $100,0 \%$ \\
\hline \multirow{2}{*}{ Total } & $\mathrm{n}$ & 31 & 09 & 40 \\
\hline & $\%$ & $77,5 \%$ & $22,5 \%$ & $100,0 \%$ \\
\hline
\end{tabular}

Fonte: Pesquisa de Campo, 2009. 
Tabela 2 - Descrição da sensibilização da equipe de enfermagem para alcance das metas dos indicadores. Montes Claros-MG (2009).

\begin{tabular}{|c|c|c|c|}
\hline Variável & Categorias & $\mathbf{n}$ & $\%$ \\
\hline $\begin{array}{l}\text { Considera a sensibilização da equipe de enfermagem para al- } \\
\text { cance das metas conforme os indicadores de processo }\end{array}$ & $\begin{array}{c}\text { Ruim } \\
\text { Regular } \\
\text { Bom } \\
\text { Muito Bom } \\
\text { Excelente }\end{array}$ & $\begin{array}{l}01 \\
10 \\
20 \\
07 \\
02\end{array}$ & $\begin{array}{c}2,5 \\
25,0 \\
50,0 \\
17,5 \\
5,0\end{array}$ \\
\hline
\end{tabular}

Fonte: Pesquisa de Campo, 2009.

Abordando especificamente as dificuldades vivenciadas pelo enfermeiro na utilização de indicadores, esse profissional pôde escolher durante a pesquisa entre mais de uma alternativa citada como dificuldade e ainda, acrescentar alguma outra ou outras não relacionadas no questionário, mas que o mesmo vivenciou como sendo um fator dificultador para utilização da ferramenta.

O instrumento da coleta de dados considerou o tempo em que os profissionais estão na instituição para analisar se havia ou não diferença, em relação às dificuldades para utilizar indicadores de processos.

O presente estudo investigou a qualidade das informações transmitidas ao enfermeiro no processo de implantação da utilização de indicadores, sendo constado que $72,5 \%$ dos enfermeiros consideraram entre boa a muito boa a qualidade das informações recebidas para se trabalhar com indicadores.

Quando abordado o enfermeiro sobre a importância da utilização de indicadores para avaliar a qualidade dos processos assistenciais, $62,5 \%$ consideraram a ferramenta extremamente importante e $27,5 \%$ muito importante; as demais categorias, pouca importância e importante, perfizeram juntas $10 \%$. Quando esse profissional foi questionado se a utilização de indicadores é capaz de melhorar as ações assistenciais da equipe de enfermagem, $57,5 \%$ consideraram que o trabalho melhora de modo extremamente importante as ações da equipe, $27,5 \%$, de modo muito importante, $12,5 \%$ de maneira importante e 2,5\% consideram de pouco importância.

Quanto à coleta de dados realizada pela equipe de enfermagem, indagou-se ao enfermeiro sobre a qualidade da coleta realizada por sua equipe, constatando-se que $47,5 \%$ a consideram de boa a muito boa qualidade, $44,7 \%$ acham que a qualidade é de regular a ruim e $7,5 \%$ não opinaram, por não terem essa experiência. Boa parte dos enfermeiros participantes da pesquisa $(52,5 \%)$ considerou a equipe de enfermagem um agente facilitador para a coleta de dados, contra $42,5 \%$ dos enfermeiros que a acharam dificultador e indiferente. Já a sensibilização da equipe para o alcance das metas pactuadas conforme os dados obtidos para contribuir com a melhoria da assistência de enfermagem Tabela 2.

\section{DISCUSSÃO}

Os dados obtidos demonstraram que a qualificação do enfermeiro relativa à Gestão da Qualidade e por Processos, que trabalham com a mensuração dos resultados assistenciais e não assistenciais dentro de uma unidade hospitalar, está inadequada. O enfermeiro deveria ter se familiarizado com o tema em sua graduação por meio das disciplinas de Administração/Gerenciamento dos Serviços de Saúde, visto que a preocupação com a Gestão de Qualidade é mundial e se originou em 1945, se intensificando no setor saúde no final da década de $1960^{(6)}$, mas o mesmo não ocorreu nas universidades formadoras dos profissionais pesquisados.

O próprio Manual Brasileiro de Acreditação Hospitalar, criado desde 1998 para se atingir padrões de qualidade dos serviços de saúde, no qual é inserida a necessidade e importância de se trabalhar com indicadores, ainda não é discutido em algumas graduações, o que demonstra que o período de dez anos é insuficiente para a mudança em determinados cursos de enfermagem ${ }^{(7)}$.

Quanto à dificuldade em utilizar indicadores para avaliar a qualidade dos resultados de processos assistenciais, constatou-se que $94,9 \%$ dos enfermeiros participantes relataram algum tipo de dificuldade ao iniciar o trabalho com essa ferramenta.

Resultado confirmado em outro estudo quando o autor coloca que apesar do desenvolvimento da utilização de indicadores nos últimos anos, ainda não foram produzidos modelos capazes de padronizar a gestão de informação nos hospitais devendo cada instituição desenvolver suas competências na utilização de indicadores ${ }^{(2)}$.

Assim, é razoável reconhecer que nem sempre o enfermeiro possui esse conhecimento, resultando em dificuldade para a utilização adequada de indicadores para mensurar a assistência de enfermagem. Essa dificuldade, entretanto, pode trazer desvantagens para a instituição, uma vez que os indicadores não são apenas uma ferramenta de controle, mas, principalmente, uma maneira sistematizada de melhor compreender e conhecer a realidade organizacional, além de analisar a validade das estratégias definidas pela empresa ${ }^{(15)}$.

Quando é revelado pela pesquisa que $77,5 \%$ dos participantes não tiveram nenhum tipo de contato teórico e/ ou prático com o tema durante a sua graduação, não sendo o conteúdo contemplado nos currículos da maior parte dos estabelecimentos de ensino, encontra-se uma das razões da dificuldade do enfermeiro em utilizar indicadores, que necessita ser urgentemente revista pelos cursos de graduação.

Esse resultado em muito se parece com o obtido em outro estudo ${ }^{(16)}$, em que, da amostra estudada, $84 \%$ nunca havia trabalhado com indicadores em outras instituições, 
demonstrando que essa equipe iniciou o aprendizado e desenvolvimento do trabalho juntamente com o hospital, o que denota a inicialização das pesquisas.

Ao se analisar especificamente as dificuldades apontadas pelos enfermeiros como fatores intervenientes na utilização de indicadores, as alternativas mais citadas foram, em ordem decrescente: acúmulo de atividades privativas do enfermeiro dentro da instituição $(61,5 \%)$, falta de conhecimento teórico e prático do tema $(46,2 \%)$, pouco conhecimento da Gestão por Processo $(43,6 \%)$, pouco envolvimento da equipe na coleta de dados $(30,8 \%)$, falta de conhecimento prático do tema $(20,5 \%)$, falta de conhecimento teórico do tema (17,9\%), falta de acompanhamento dos gestores durante a implantação da utilização de indicadores (12,8\%).

Com relação ao acúmulo das atividades que a instituição determina como sendo de realização exclusiva do enfermeiro dentro do hospital, apontadas como principal causa dificultadora de se trabalhar com indicadores, é preciso refletir sobre essas atividades e analisá-las criticamente reorganizando o processo de trabalho desse profissional dentro de suas unidades de trabalho. Pois, essas atividades estão em desacordo com as privativas da regulamentação legal da categoria profissional e torna custosa a ação do enfermeiro gerenciar os indicadores de seu setor, tanto na coleta, análise e divulgação dos mesmos para a sua equipe de trabalho.

Abordando a dificuldade de se trabalhar com indicadores devido à falta de conhecimento teórico e prático do tema, segunda maior dificuldade entre os enfermeiros participantes do estudo, retoma a discussão anterior sobre a formação acadêmica dos profissionais recém-formados, mas também aponta para a necessidade constante do aperfeiçoamento e atualização daqueles já inseridos no mercado de trabalho. Afinal, é o mercado de trabalho que exige do enfermeiro a sua capacitação para trabalhar com ferramentas administrativas e práticas do gerenciamento de recursos para se alcançar às metas da instituição ${ }^{(3)}$.

A terceira maior dificuldade para utilização de indicadores foi o pouco conhecimento teórico sobre a Gestão por Processo, sendo as causas desse, os mesmos itens acima abordados sobre o processo de formação e aperfeiçoamento profissional. Aperfeiçoamento esse que perpassa a utilização de ferramentas administrativas, aqui em voga a gestão por processos, eleita pela Acreditação Hospitalar, por sua eficiência na busca contínua pela qualidade da assistência prestada ao cliente, sempre focando os processos ${ }^{(2)}$.

A quarta maior dificuldade em utilizar indicadores para melhoria da qualidade da assistência apontada pelos enfermeiros foi o pouco envolvimento da equipe na coleta de dados, o que já era de se esperar, até mesmo os administradores hospitalares possuem dificuldades para trabalhar com essa ferramenta ${ }^{(5)}$. Isso não podia ser diferente com a equipe de enfermagem, principalmente com auxiliares e técnicos, pois, até o momento, só lhes era cobrado o fazer, o executar tarefas sem nenhuma visão do impacto que elas ocasionavam na qualidade da assistência aos clientes. Trata-se da mudança de cultura e filosofia de toda uma vida de trabalho baseada no cuidar sem se preocupar com a importância do administrar e participar dessa administração definindo metas e ações para alcançá-las.

Por fim, outra dificuldade apontada nos resultados do estudo foi a falta de acompanhamento dos gestores da instituição durante a implantação da utilização de indicadores demonstrando que apenas o conhecimento teórico sobre o assunto não foi suficiente para solucionar dúvidas e minimizarem as dificuldades, afinal, existe um consenso entre os administradores da complexidade de se lidar com indicadores, existindo dificuldades desde a escolha do sistema de avaliação até os tipos de indicadores mais adequados a realidade institucional ${ }^{(5)}$. Porém, ao se analisar a opinião dos pesquisados sobre as orientações recebidas à época da implantação ou a sua inserção na instituição para utilização dos indicadores, 72,5\% consideraram que a orientação foi de boa a muito boa.

A pesquisa teve a preocupação de verificar, junto aos enfermeiros que participaram do estudo, o que os mesmos achavam da importância da ferramenta para avaliar a qualidade dos processos assistenciais de sua equipe de trabalho, mesmo diante das dificuldades em utilizá-la, sendo o resultado encontrado extremamente positivo, visto que $97,5 \%$ consideraram o uso de indicadores de importante a extremamente importante.

Vê-se assim, uma mudança de cultura por detecção da importância de uma mudança no fazer por fazer, mas fazer com planejamento e visão crítica de todo o processo assistencial, pois também 97,5\% dos participantes da pesquisa acreditam que a utilização de indicadores é capaz de melhorar as ações assistenciais da equipe de enfermagem. Ideia reforçada em outros estudos, que afirmam existir a necessidade de cada instituição desenvolver sua capacidade para incorporar e utilizar indicadores, obtidos por meio de uma coleta de dados segura e com qualidade ${ }^{(4)}$.

Outro fator pesquisado foi a sensibilização da equipe para o alcance das metas pactuadas a partir dos indicadores, observando-se que a grande maioria dos enfermeiros $(67,5 \%)$ considerou a equipe técnica de enfermagem sensibilizada de forma boa a muito boa para o alcance das metas. O que é muito importante para se atingir a excelência desejada dentro da instituição, pois é necessário compreender as pessoas e suas percepções para depois discutir a qualidade, enquanto produto do trabalho dessas, e nunca se esquecendo de valorizá-las e envolvê-las no processo de mudança capaz de alcançar a meIhoria da qualidade dos serviços ${ }^{(17)}$. Afinal, o envolvimento dos colaboradores da instituição é a força que viabiliza o alcance de resultados satisfatórios dos processos de trabalho e, o que é mais importante, garante a prestação de uma assistência de enfermagem segura, com qualidade e humanizada.

\section{CONCLUSÕES}

A análise dos resultados permitiu afirmar que 94,9\% dos enfermeiros participantes relataram algum tipo de dificuldade ao iniciar o trabalho com essa ferramenta, sendo uma das principais causas relacionadas a essa dificuldade, o acumulo de atividades privativas do enfermeiro dentro da Santa Casa, o que demanda a necessidade de se refletir sobre as demais 
atividades desse profissional e, se necessário, reorganizar seu processo de trabalho. É preciso, também, citar o fato de alguns cursos de graduação em enfermagem precisarem se atualizar quanto ao assunto, pois, em dez anos, desde a criação da ONA até os dias atuais, a mudança foi insuficiente para as exigências do mercado, que cada vez avança mais.

Outro ponto que necessita ser destacado é o de não existir no mercado modelos prontos para se trabalhar com indicadores, bem como enfermeiros formados com conhecimento suficiente para trabalhar com indicadores de processo, já que o tema não é contemplado nos currículos da grande parte das instituições de ensino. Fato que necessita causar inquietação para alterações nos cursos de graduação.
Porém, comprovou-se que apesar das dificuldades encontradas em utilizar indicadores, a grande maioria dos enfermeiros consideraram o uso dessa ferramenta de importante a extremamente importante, o que demonstra a conscientização desse profissional das exigências do mercado e da necessidade de atualizações.

Mas, o mais importante foi a comprovação do envolvimento dos colaboradores da equipe de enfermagem com o alcance das metas pactuadas por meio dos indicadores, fazendo com que a excelência da assistência seja alcançada mais facilmente, apesar dos obstáculos que, com certeza, ainda virão. Havendo vontade, tudo é possível de ser alcançado e realizado, e a vontade já existe instituição.

\section{REFERÊNCIAS}

1. Donabedian A. The role of outcomes in quality assessment and assurance. QRB Qual Rev Bull 1992;18 (11):356-360.

2. Oliveira SBO. Gestão por processos: fundamentos, técnicas e modelos de implementação: foco no sistema de gestão de qualidade com base na ISO 9000. Rio de Janeiro: Qualitymark; 2006.

3. Sanna MC. Os processos de trabalho em enfermagem. Rev Bras Enferm 2007;60(2):221-224.

4. Schout DN, Novaes HMD. Do registro ao indicador: gestão da produção da informação assistencial nos hospitais. Ciên Saúde Coletiva 2007;12(4):935-944.

5. Escrivão Junior A. Uso da informação na gestão de hospitais públicos. Ciên Saúde Coletiva 2007:12(3):655-666.

6. D'Innocenzo M, Feldman LB, Fazenda NRR, Helito RAB, Ruthes RM. Indicadores, Auditorias, Certificações - Ferramentas de qualidade para Gestão em Saúde. São Paulo: Martinari; 2006.

7. Organização Nacional de Acreditação [homepage na internet]. [acesso em 30 mar 2008]. Disponível em: http://www.ona.org.br/site/internal_institucional.jsp. pagesite $=$ histórico .

8. Vituri DW, Matsuda LM. Validação de conteúdo de indicadores de qualidade para avaliação do cuidado de enfermagem. Rev Esc Enferm USP 2009;43(2):429-437.

9. Ministério da Defesa do Exército Brasileiro. Programa Excelência Gerencial: Indicadores de Desempenho - Sistema de Medição de Desempenho Organizacional. Brasília, DF: Ministério da Defesa do Exército Brasileiro; 2004.

10. Bittar OJNV. Indicadores de quantidade e qualidade em saúde. Rev Adm Saúde 2001;3(12):21-28.

11. Zanon U. Qualidade da assistência médico-hospitalar: conceito, avaliação e discussão dos Indicadores de qualidade. Rio de Janeiro: MEDSI; 2001.

12. Chiavenato I. Teoria Geral da Administração. São Paulo: Makron Books, 1993.

13. França JL. Manual para normalização de publicações técnico-científicas. 5. ed. Belo Horizonte: UFMG; 2001.

14. Duarte SV, Furtado MS. Manual para Elaboração de Monografias e Projetos de Pesquisa. Montes Claros: Unimontes; 2002.

15. Kiyan FM. Proposta para desenvolvimento de indicadores de desempenho como suporte estratégico. São Carlos. Dissertação [Mestrado em Engenharia de Produção] - Escola de Engenharia de São Carlos da USP; 2001.

16. Franco JN, Barros BPA, Vaidotas M, D'innocenzo M. Percepção dos enfermeiros sobre os resultados dos indicadores de qualidade na melhoria da prática assistencial. Rev Bras Enferm 2010;63(5):806-810.

17. Cunha ICKO, Feldman LB. Avaliação dos serviços de enfermagem: identificação dos critérios de processo dos programas de acreditação hospitalar. Rev Bras Enferm 2005;58(1):65-69. 